\title{
Tolerance of New Introgressive Hybrid and Backcross Forms Pathogenic Micromitisms (Verticillium dahliae Kleb and Fusarium oxysporum f.sp. vasinfectum)
}

\author{
B. A. Sirojidinov'1, A. Abdullayev'1, A. G. Sherimbetov², A. A. Narimanov², B. A. Omonov² \\ ${ }^{1}$ Institute of Botany, Uzbek Academy of Science, Tashkent, Uzbekistan \\ ${ }^{2}$ Institute of Genetics and Plant Experimental Biology, Uzbek Academy of Science, Tashkent, Uzbekistan \\ Email: behzod_arabdjonovich@mail.ru
}

How to cite this paper: Sirojidinov, B.A., Abdullayev, A., Sherimbetov, A.G., Narimanov, A.A. and Omonov, B.A. (2018) Tolerance of New Introgressive Hybrid and Backcross Forms Pathogenic Micromitisms (Verticillium dahliae Kleb and Fusarium oxysporum f.sp. vasinfectum). American Journal of Plant Sciences, 9, 1308-1320. https://doi.org/10.4236/ajps.2018.96096

Received: March 27, 2018

Accepted: May 27, 2018

Published: May 30, 2018

Copyright $\odot 2018$ by authors and Scientific Research Publishing Inc. This work is licensed under the Creative Commons Attribution International License (CC BY 4.0).

http://creativecommons.org/licenses/by/4.0/ c) (i) Open Access

\begin{abstract}
The article is based on the use of experimental polyploidy method, with the introduction of new introgressive hybrid forms combining several species genotype with pathogenic Verticillium dahliae Kleb. and the effects of mycotoxins separated from the Fusarium oxysporum f.sp. vasinfectum micromicette on the yield of plant seeds. New artificial complex hypertension forms based on experimental polyploidy Verticillium dahliae Kleb. and Fusarium oxysporum f.sp. vasinfectum combine the potential of resistance to mycotoxins separated from microcrystals, making a tremendous contribution to the selection of new varieties and to the effectiveness of selection as a result of the use of genetic selective research as genetic-selective genetic-selector studies.
\end{abstract}

\section{Keywords}

Verticillium dahliae Kleb., Fusarium oxysporum f.sp. vasinfectum, Cotton, Mycotoxin, Experimental Polyploidy

\section{Introduction}

Currently, one of the major areas of agriculture in the world is focusing on the improvement of the genetic capacity of valuable crops of cultivated crops through the use of cotton (Gossypium L.) crop, which is a genetically resistant source of resistant diseases and pests and wild ancestors of agricultural crops. One of the pressing issues is the expansion of cotton-fiber orientation and the 
creation of highly productive cotton varieties with high competitiveness and high quality in the world.

As known, the most common pathogenic Verticillium and Fusarium species in the microorganisms in the soil have a negative effect on the disease of one year, two years and perennial plants [1] [2].

Sherimbetov A.G. [3] studied the species of G. hirsutum L. on the varieties of samples in their varieties, which is separated from fungi belonging to Fusarium family. As a result of the research, the 576,601,656 strains of the $F$. oxysporum strains isolated from the fucarial inflammation in the Bukhara region were found to be $8.0 \%-35.0 \%$, and 6 of Bukhara-16.0\% - 31.0\% which had a negative impact on the decline. The 595 strains isolated from the soil have detected the negative effects of AN-Bayaut- 2 and Bukhara 6 on $100.0 \%$ seeds degradation.

Participation in the Gossypium L. tetraploidal and diploidal species involves the use of experimental polyploidy techniques to provide valuable economic and agricultural diseases (gomosis, fuzariosis, fuzariosis, and verticalisation), resistant to insecticides. And the results of research by foreign scientists have been applied to genetics and selective selection [4]-[11]. Specifically Australian varieties $(2 n=26)$ have valuable properties. In particular, insufficient gossypol glands in the seeds are consumed by humans and animals as potential source of food, pest infestation (Aphis gossupii Glov., Acari Leach), diseases (Fusarium, Verticillium), low temperature and abiotic stress drought are resistant to factors [12] [13] [14] [15]. G. arboreum diploidal type $(2 \mathrm{n}=26)$ was identified as pests (Apolygus lucorum) [16], disease (Verticillium dahliae, Fusarium oxysporum) [17] [18] [19] and drought resistance [20].

S.S. Kanash [21] [22] has studied instrumental solving many of the theoretical and practical questions facing genetic and selection. It has been shown that $G$. hirsutum L. and G. herbaceum L. species are resistant to germose (Xanthomonas malvacearum Dows.), Resistant to the hybrid of G. barbbadense L. and G. arboreum L. on the basis of hybridisation by S-3802 resistant to fusarium (Fuzarium oxysporum $f$. vasinfectum $N D$ ) and has a close proximity to the G. barbbadense species of $G$. arboreum species on the silkiness, elasticity and aging of the fiber.

Specimen species of $G$. herbaceum L. and $G$. arboreum L. were used as donor species in intersection of tetraploid varieties, especially for disease and insect resistance [23].

\section{Materials and Methods}

The first source of research is the new introgressive hybrid forms [24], which combine genotype with a number of species on the basis of the experimental polyploidy method and Verticillium dahliae Kleb. and Fusarium oxysporum f.sp. vasinfectum (obtained from the Collection of Phytopathogenic Microorganisms-a Unique Scientific Object of the Institute of Genetics and Plant Experimental Biology of the Academy of Sciences of Uzbekistan). 
The fungal specimens were grown in $100 \mathrm{ml}$ of the Chapek-Doksa feeding medium for 15 days in $250 \mathrm{ml}$ tubes at a temperature of $25^{\circ} \mathrm{C}-270^{\circ} \mathrm{C}$ (Figure $1(\mathrm{a})$ ). After the cultivation process was completed, filtered to separate the mycelium from the feed medium (Figure 1(b)).

The effects of toxins in the cultured fluid of fungi were tested for 30 seeds.

Seeds of 30 seeds were screened for cultured fluid in the fungus for one day. Seeds of control were sown in Chapek-Doksa food and distilled water (Figure $1(\mathrm{c})$ ). Sprinkled seeds were placed in a sun-dried camera with a temperature of $18^{\circ} \mathrm{C}$ to $200^{\circ} \mathrm{C}$ (Figure 1(d) and Figure $1(\mathrm{e})$ ) to observe the rate of flour in a 7 to 10-day moist cell in a Petri dish. On the tenth day of the experiment, the rate of seeding, the root and the length of the seeds were measured (Figure 1(f)).

Characteristics of mitotoxin cultivation of fungi are reduced by the reduction of reproductive tissue, reduction of root and stomach growth by the following formula:

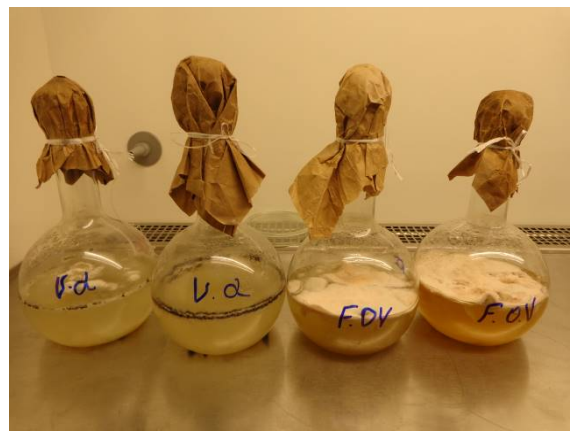

(a)

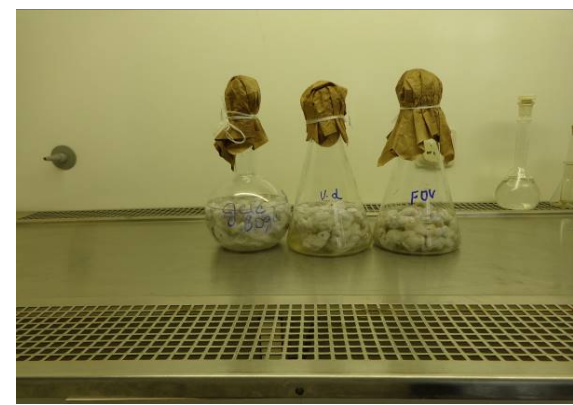

(c)

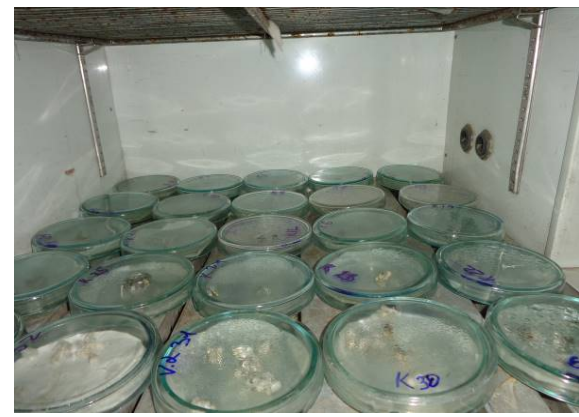

(e)

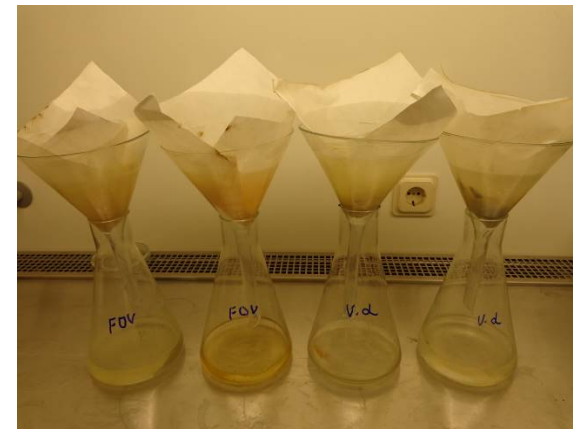

(b)

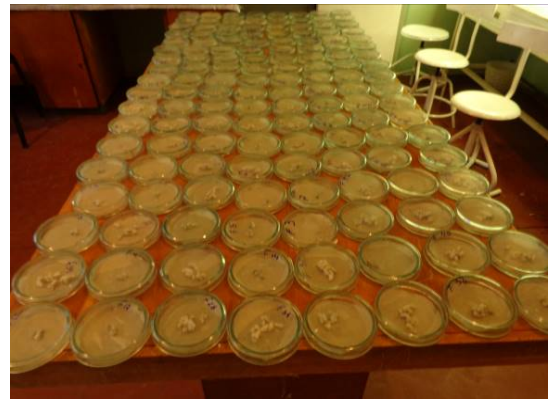

(d)

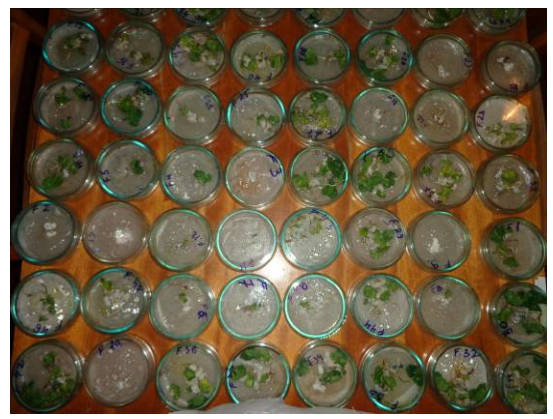

(f)

Figure 1. Research methods. 


$$
T=100 \%-(L / L k \cdot 100)
$$

Depending on the pathogenicity of studied plants, it is divided into the following groups:

Strong resistant-no more than $0.0 \%-30.0 \%$ seeds.

Low-resistant-31.0\% - 50.0\% without seeds.

Medium-resistant-51.0\% - 70.0\% without seeds.

Strongly unstable-71.0\% - $100.0 \%$ of seeds did not sprout.

\section{Results}

During the research, the level of influence of mycotoxins on the yield of plant seeds separated from microcucetes of Verticillium dahliae Kleb., Fusarium oxysporum f.sp. vasinfectum was investigated, using new experimental hypertrophy forms combining genotype with the use of experimental polyploidy method. It should be noted that the introgressive hybrid forms of studied plants showed that the tolerance of plant seeds was $100.0 \%$ (Table 1) $\mathrm{F}_{2} \mathrm{C}$ G. hirsutum ssp. euhirsutum "Kelajak" $\times$ (ssp. nanking (white fiber $) \times G$. nelsonii) hybrids combination. The low strength of mycelium (50.0\%) against mycotoxins of Verticillium dahliae and Fusarium oxysporum f.sp. vasinfectum was observed (Figure 2).

$\mathrm{F}_{2} \mathrm{C}$ G. hirsutum ssp. euhirsutum "Namangan 77 " $\times$ (ssp. obtusifolium, indicum $\times$ G. australe) hybrid combination with mycelium from Verticillium dahliae was $50.0 \%$. In this study, in combination with hybrid, the resistance of the mycotoxins from the Fusarium oxysporum f.sp. vasinfectum microcrycate was strongly determined (75.0\% of the seeds did not grow).

$\mathrm{F}_{2} \mathrm{~B}_{1} \mathrm{C}$ Kelajak $\times[$ Kelajak $\times$ (ssp. nanking (with white fiber) $\times$ G. nelsonii) $]$ backcross hybrid combination of bacterial bicolor Verticillium dahliae tolerance was $90.0 \%$. The effect of mycotoxins separated from the fusarium oxysporum f.sp. vasinfectum microcrystine was $50.0 \%$. In the future combinations of bacterium bacteria, Verticillium dahliae, the mycotoxin effect is the result of plant $25.0 \%$ to $30.0 \%$ of the seeds are resistant to frostbite. In the combination of $\mathrm{F}_{2} \mathrm{~B}_{1} \mathrm{C}$ Наманган $77 \times[$ Наманган $77 \times($ ssp. obtusifolium var. indicum $\times$

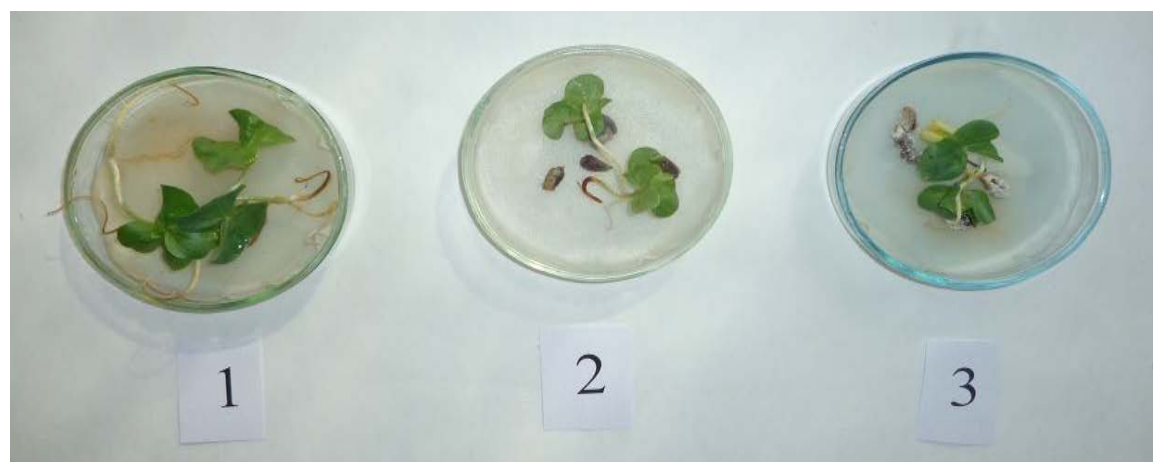

Explanation: 1. Control; 2. Verticillium dahliae, 3. Fusarium oxysporum f.sp. vasinfectum

Figure 2. $\mathrm{F}_{2}$ Kelajak $\times($ ssp. nanking $($ white fiber $) \times$ G. nelsonii $)$. 
Table 1. Introgressive hybrid forms Verticillium dahliae Kleb., Fusarium oxysporum f.sp. vasinfectum degree of influence of mycotoxins on microorganisms on bacterial seed tolerance.

\begin{tabular}{|c|c|c|c|c|c|c|c|}
\hline \multirow{2}{*}{ № } & \multirow{2}{*}{ Hybrid and backcross combinations } & \multicolumn{2}{|c|}{ Control } & \multicolumn{2}{|c|}{$\begin{array}{l}\text { Verticillium } \\
\text { dahliae Kleb. }\end{array}$} & \multicolumn{2}{|c|}{$\begin{array}{c}\text { Fusarium } \\
\text { oxysporum f.sp. } \\
\text { vasinfectum }\end{array}$} \\
\hline & & $\begin{array}{l}\text { Grown } \\
\text { Seeds } \\
\%\end{array}$ & $\begin{array}{c}\text { Non } \\
\text { grown } \\
\text { seeds \% }\end{array}$ & $\begin{array}{l}\text { Grown } \\
\text { Seeds } \\
\%\end{array}$ & $\begin{array}{c}\text { Non } \\
\text { grown } \\
\text { seeds \% }\end{array}$ & $\begin{array}{l}\text { Grown } \\
\text { Seeds } \\
\%\end{array}$ & $\begin{array}{c}\text { Non } \\
\text { grown } \\
\text { seeds \% }\end{array}$ \\
\hline \multicolumn{8}{|c|}{$F_{2}$ hybrids } \\
\hline 1 & Namangan $77 \times($ ssp. obtusifolium var. indicum $\times$ G. australe $)$ & 100.0 & 0.0 & 50.0 & 50.0 & 25.0 & 75.0 \\
\hline 2 & Kelajak $\times($ ssp. nanking $($ with white fiber $) \times G$. nelsonii $)$ & 100.0 & 0.0 & 50.0 & 50.0 & 50.0 & 50.0 \\
\hline \multicolumn{8}{|c|}{$F_{2} B_{1} C$ backcross hybrids } \\
\hline 3 & Namangan $77 \times$ [Namangan $77 \times($ ssp. obtusifolium var. indicum $\times$ G. australe $)]$ & 100.0 & 0.0 & 25.0 & 75.0 & 0.0 & 100.0 \\
\hline 4 & [Namangan $77 \times($ ssp. obtusifolium var. indicum $\times$ G. australe $)] \times$ Namangan 77 & 100.0 & 0.0 & 0.0 & 100.0 & 25.0 & 75.0 \\
\hline 5 & Kelajak $\times[$ Kelajak $\times($ ssp. nanking $($ with white fiber $) \times G$. nelsonii $)]$ & 100.0 & 0.0 & 90.0 & 10.0 & 50.0 & 50.0 \\
\hline 6 & {$[$ Kelajak $\times($ ssp. nanking $($ with white fiber $) \times G$. nelsonii $)] \times$ Kelajak } & 100.0 & 0.0 & 30.0 & 75.0 & 0.0 & 100.0 \\
\hline \multicolumn{8}{|c|}{$F_{3}$ hybrids } \\
\hline 7 & Namangan $77 \times($ ssp. obtusifolium var. indicum $\times$ G. australe) $($ Family 1$)$ & 100.0 & 0.0 & 80.0 & 20.0 & 50.0 & 50.0 \\
\hline 8 & Namangan $77 \times($ ssp. obtusifolium var. indicum $\times$ G. australe) $($ Family 2$)$ & 100.0 & 0.0 & 70.0 & 30.0 & 90.0 & 10.0 \\
\hline 9 & Namangan $77 \times($ ssp. obtusifolium var. indicum $\times$ G. australe) $($ Family 3$)$ & 100.0 & 0.0 & 70.0 & 30.0 & 60.0 & 40.0 \\
\hline 10 & Namangan $77 \times($ ssp. obtusifolium var. indicum $\times$ G. australe) $($ Family 4$)$ & 100.0 & 0.0 & 0.0 & 100.0 & 50.0 & 50.0 \\
\hline 11 & Kelajak $\times($ ssp. nanking $($ with white fiber $) \times G$. nelsoniit $)($ Family 1$)$ & 100.0 & 0.0 & 50.0 & 50.0 & 55.0 & 45.0 \\
\hline 12 & Kelajak $\times($ ssp. nanking $($ with white fiber $) \times G$. nelsonii $)($ Family 2$)$ & 100.0 & 0.0 & 66.7 & 33.3 & 80.0 & 20.0 \\
\hline 13 & Kelajak $\times($ ssp. nanking $($ with white fiber $) \times G$. nelsonii $)($ Family 3$)$ & 100.0 & 0.0 & 90.0 & 10.0 & 50.0 & 50.0 \\
\hline 14 & Kelajak $\times($ ssp. nanking $($ with white fiber $) \times G$. nelsonii $)($ Family 4$)$ & 100.0 & 0.0 & 90.0 & 10.0 & 30.0 & 70.0 \\
\hline \multicolumn{8}{|c|}{$F_{3} B_{1} C$ backcross hybrids } \\
\hline 15 & $\begin{array}{c}\text { Namangan } 77 \times \text { [Namangan } 77 \times(\text { ssp. obtusifolium var. indicum } \times \text { G. australe })] \\
(\text { Family } 1)\end{array}$ & 100.0 & 0.0 & 25.0 & 75.0 & 0.0 & 100.0 \\
\hline 16 & $\begin{array}{c}\text { Namangan } 77 \times \text { [Namangan } 77 \times(\text { ssp. obtusifolium var. indicum } \times \text { G. australe })] \\
(\text { Family } 2)\end{array}$ & 100.0 & 0.0 & 95.0 & 5.0 & 0.0 & 100.0 \\
\hline 17 & $\begin{array}{c}\text { Namangan } 77 \times \text { [Namangan } 77 \times(\text { ssp. obtusifolium var. indicum } \times \text { G. australe })] \\
(\text { Family } 3)\end{array}$ & 100.0 & 0.0 & 50.0 & 50.0 & 0.0 & 100.0 \\
\hline 18 & $\begin{array}{c}\text { Namangan } 77 \times[\text { Namangan } 77 \times(\text { ssp. obtusifolium var. indicum } \times \text { G. australe })] \\
(\text { Family } 4)\end{array}$ & 100.0 & 0.0 & 0.0 & 100.0 & 50.0 & 50.0 \\
\hline 19 & $\begin{array}{l}{[\text { Namangan } 77 \times(\text { ssp. obtusifolium var. indicum } \times \text { G. australe })] \times \text { Namangan } 77} \\
(\text { Family } 1)\end{array}$ & 100.0 & 0.0 & 0.0 & 100.0 & 0.0 & 100.0 \\
\hline 20 & $\begin{array}{c}\text { [Namangan } 77 \times(\text { ssp. obtusifolium var. indicum } \times \text { G. australe })] \times \text { Namangan } 77 \\
(\text { Family } 2)\end{array}$ & 100.0 & 0.0 & 0.0 & 100.0 & 95.0 & 5.0 \\
\hline 21 & $\begin{array}{l}{[\text { Namangan } 77 \times(\text { ssp. obtusifolium var. indicum } \times \text { G. australe })] \times \text { Namangan } 77} \\
(\text { Family } 3)\end{array}$ & 100.0 & 0.0 & 66.7 & 33.3 & 95.0 & 5.0 \\
\hline 22 & $\begin{array}{c}\text { [Namangan } 77 \times(\text { ssp. obtusifolium var. indicum } \times \text { G. australe })] \times \text { Namangan } 77 \\
(\text { Family } 4)\end{array}$ & 100.0 & 0.0 & 96.0 & 4.0 & 96.0 & 4.0 \\
\hline
\end{tabular}




\section{Continued}

\begin{tabular}{|c|c|c|c|c|c|c|c|}
\hline 23 & Kelajak $\times[$ Kelajak $\times($ ssp. nanking $($ with white fiber $) \times G$. nelsonii $)]$ (Family 1$)$ & 100.0 & 0.0 & 25.0 & 75.0 & 25.0 & 75.0 \\
\hline 24 & Kelajak $\times[$ Kelajak $\times($ ssp. nanking $($ with white fiber $) \times G$. nelsonii) $]$ (Family 2$)$ & 100.0 & 0.0 & 33.3 & 66.7 & 95.0 & 5.0 \\
\hline 25 & Kelajak $\times[$ Kelajak $\times($ ssp. nanking $($ with white fiber $) \times G$. nelsonii $)]($ Family 3$)$ & 100.0 & 0.0 & 96.0 & 4.0 & 95.0 & 5.0 \\
\hline 26 & Kelajak $\times[$ Kelajak $\times($ ssp. nanking $($ with white fiber $) \times G$. nelsonii $)]($ Family 4$)$ & 100.0 & 0.0 & 50.0 & 50.0 & 95.0 & 5.0 \\
\hline 27 & $[$ Kelajak $\times($ ssp. nanking $($ with white fiber $) \times G$. nelsonii $)] \times$ Kelajak (Family 1$)$ & 100.0 & 0.0 & 50.0 & 50.0 & 25.0 & 75.0 \\
\hline 28 & $[$ Kelajak $\times($ ssp. nanking $($ with white fiber $) \times$ G. nelsonii $)] \times$ Kelajak (Family 2$)$ & 100.0 & 0.0 & 25.0 & 75.0 & 25.0 & 75.0 \\
\hline 29 & $[$ Kelajak $\times($ ssp. nanking $($ with white fiber $) \times$ G. nelsonii $)] \times$ Kelajak (Family 3$)$ & 100.0 & 0.0 & 0.0 & 100.0 & 0.0 & 100.0 \\
\hline 30 & $[$ Kelajak $\times($ ssp. nanking $($ with white fiber $) \times$ G. nelsonii $)] \times$ Kelajak (Family 4$)$ & 100.0 & 0.0 & 95.0 & 5.0 & 0.0 & 100.0 \\
\hline \multicolumn{8}{|c|}{$F_{4}$ hybrids } \\
\hline 31 & Namangan $77 \times($ ssp. obtusifolium var. indicum $\times$ G. australe $)($ Family 1$)$ & 100.0 & 0.0 & 70.0 & 30.0 & 70.0 & 30.0 \\
\hline 32 & Namangan $77 \times($ ssp. obtusifolium var. indicum $\times$ G. australe) $($ Family 2$)$ & 100.0 & 0.0 & 95.0 & 5.0 & 80.0 & 20.0 \\
\hline 33 & Namangan $77 \times($ ssp. obtusifolium var. indicum $\times$ G. australe) $($ Family 3$)$ & 100.0 & 0.0 & 95.0 & 5.0 & 90.0 & 10.0 \\
\hline 34 & Namangan $77 \times($ ssp. obtusifolium var. indicum $\times$ G. australe $)($ Family 4$)$ & 100.0 & 0.0 & 70.0 & 30.0 & 70.0 & 30.0 \\
\hline 35 & Kelajak $\times($ ssp. nanking $($ with white fiber $) \times G$. nelsonii) (Family 1$)$ & 100.0 & 0.0 & 50.0 & 50.0 & 60.0 & 40.0 \\
\hline 36 & Kelajak $\times($ ssp. nanking $($ with white fiber $) \times G$. nelsonii) $($ Family 2$)$ & 100.0 & 0.0 & 90.0 & 10.0 & 90.0 & 10.0 \\
\hline 37 & Kelajak $\times($ ssp. nanking $($ with white fiber $) \times G$. nelsonii) (Family 3$)$ & 100.0 & 0.0 & 95.0 & 5.0 & 80.0 & 20.0 \\
\hline 38 & Kelajak $\times($ ssp. nanking $($ with white fiber $) \times G$. nelsonii) $($ Family 4$)$ & 100.0 & 0.0 & 96.0 & 4.0 & 96.0 & 4.0 \\
\hline
\end{tabular}

G.australe)], [Kelajak $\times($ ssp. nanking $($ with white fiber $) \times$ G. nelsonii) $] \times$ Kelajak the Verticillium dahliae did not show any suture toxic effects. It should be noted, however, that $\mathrm{F}_{2} \mathrm{~B}_{1} \mathrm{C}$ [Namangan $77 \times$ (ssp. obtusifolium var. indicum $\times$ G. australe)] $\times$ Namangan 77 coincidental bicuspid combination Fusarium oxysporum f.sp. vasinfectum has a tolerance of $25.0 \%$ (Figure 3 ).

Also, $\mathrm{F}_{2} \mathrm{~B}_{1} \mathrm{C}$ Namangan $77 \times[$ Namangan $77 \times($ ssp. obtusifolium var. indicum $\times$ G. australe)], [Kelajak $\times($ ssp. nanking $($ with white fiber $) \times$ G. nelsonii $)] \times \mathrm{Ke}-$ lajak in the future backcross hybrid combinations Fusarium oxysporum f.sp. vaginfectum the effect of mycotoxins separated from the micromycetes was recorded in the level of high durability (100.0\% of the seeds did not grow).

The effect of mycotoxins on the yield of plant seeds separated from micromieces by the family of Feminine Combine "Family 1", "Family 3" Verticillium dahliae, Fusarium oxysporum f.sp. vasinfectum with combination of hybrid combination of $\mathrm{F}_{3} \mathrm{C}$ Наманган $77 \times$ (ssp. obtusifolium var. indicum $\times$ G. australe) low and moderately resistant position. The $\mathrm{F}_{3} \mathrm{C}$ Namangan $77 \times$ (ssp. $o b$ tusifolium var. indicum $\times$ G. australe) hybrid combination of "Family 4" Verticillium dahliae mycotoxins has shown strong resistance. Fusarium oxysporum f.sp. vasinfectum has a tolerance of $50.0 \% . \mathrm{F}_{3} \mathrm{C}$ Namangan $77 \times(\mathrm{ssp}$. obtusifolium var. indicum $\times$ G. australe) in "Family 2", Verticillium dahliae tolerance was $70.0 \%$, and Fusarium oxysporum f.sp. vasinfectum caused mycotoxins by $90.0 \%$ (Figure 4). The fertility of the seeds was $50.0 \%-66.7 \%$ due to the effect of mycotoxin of Verticillium on the family of $\mathrm{F}_{3} \mathrm{C}$ Kelajak $\times$ (ssp. nanking (with white 


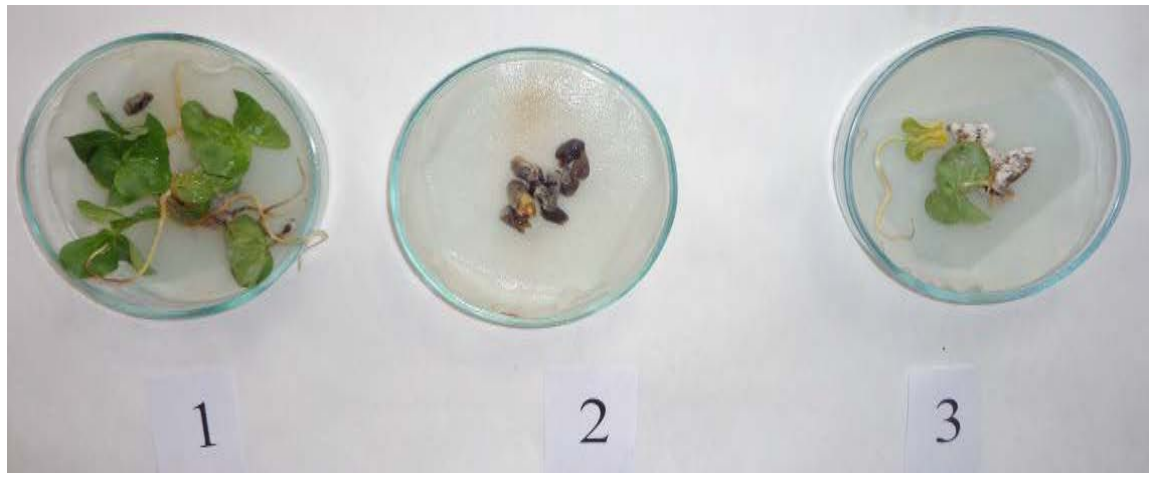

Explanation: 1. Control; 2. Verticillium dahliae, 3. Fusarium oxysporum f.sp. vasinfectum

Figure 3. $\mathrm{F}_{2} \mathrm{~B}_{1} \mathrm{C}[$ Namangan $77 \times($ ssp. obtusifolium var. indicum $\times$ G. australe $)] \times \mathrm{Na}-$ mangan 77.

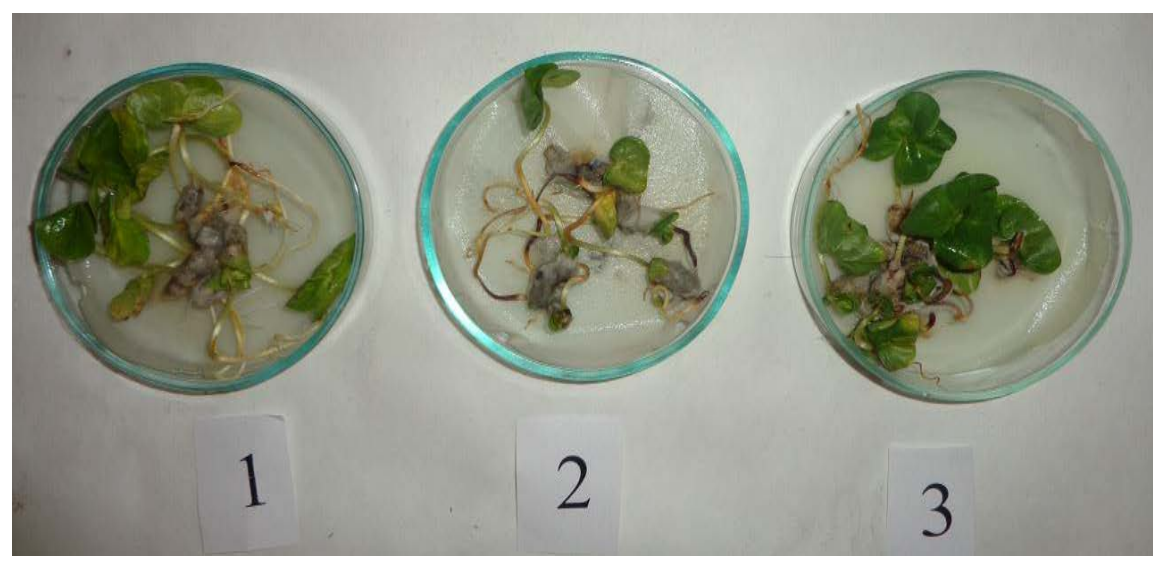

Explanation: 1. Control; 2. Verticillium dahliae, 3. Fusarium oxysporum f.sp. vasinfectum

Figure 4. $\mathrm{F}_{3} \mathrm{C}$ Namangan $77 \times($ ssp. obtusifolium var. indicum $\times$ G. australe) "Family 2".

fiber) $\times$ G.nelsonii) "Family 1", "Family 2". The $\mathrm{F}_{3} \mathrm{C}$ Kelajak $\times$ (ssp. nanking (white fiber) G. nelsonii) "Family 3" and "Family 4" were found to be $90.0 \%$ stronger than the Verticillium dahliae mycotoxin effect.

The $\mathrm{F}_{3} \mathrm{C}$ Kelajak $\times$ (ssp. nanking (white fiber) $\times$ G.nelsonii) was found to be moderately resistant to Fusarium oxysporum f.sp. vasinfectum in "Family 1", "Family 3" combination. This hybrid was recorded in the "Family 2" and "Family 4" combinations as relatively high (80,0\%).

Fumigation of germs of plant seeds was $95.0 \%$ due to mycotoxins separated from the Verticillium dahliae microscope in "Family 2" of backcross hybrid combinations of $\mathrm{F}_{3} \mathrm{~B}_{1} \mathrm{C}$ Namangan $77 \times$ [Namangan $77 \times$ (ssp. obtusifolium var. indicum $\times$ G.australe) $]$ (Figure 5(a)). $\mathrm{F}_{3} \mathrm{~B}_{1} \mathrm{C}$ Namangan $77 \times[$ Namangan $77 \times$ (ssp. obtusifolium var. indicum $\times$ G.australe)] in family "Family 1", "Family 3", Verticillium dahliae tolerance was $25.0 \%-50.0 \%$ mycotoxin resistance was found in "Family 4". Foci of uptake of seeds under the influence of mycotoxins separated from the Fusarium oxysporum f.sp. vasinfectum microscopy of "Family 4" was found to be $50.0 \%$ in the backcross hybrid combination of $\mathrm{F}_{3} \mathrm{~B}_{1} \mathrm{C} \mathrm{Na-}$ mangan $77 \times[$ Namangan $77 \times($ ssp. obtusifolium var. indicum $\times$ G.australe $)]$. 


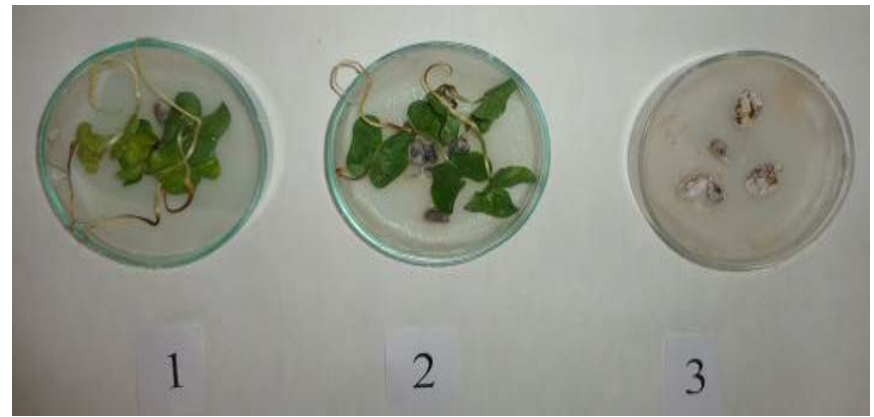

(a)

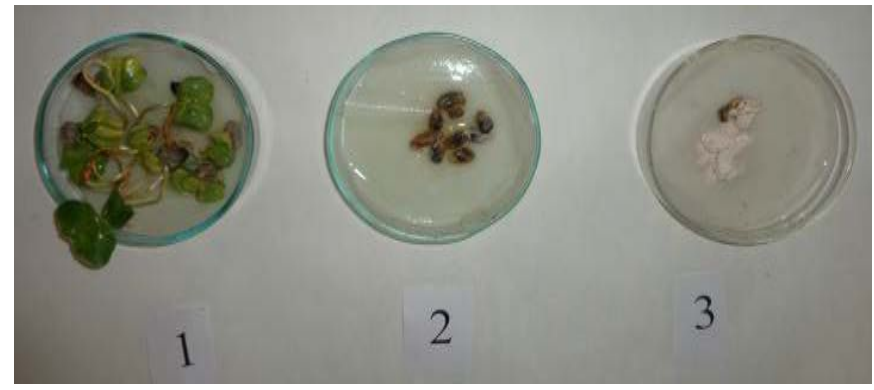

(b)

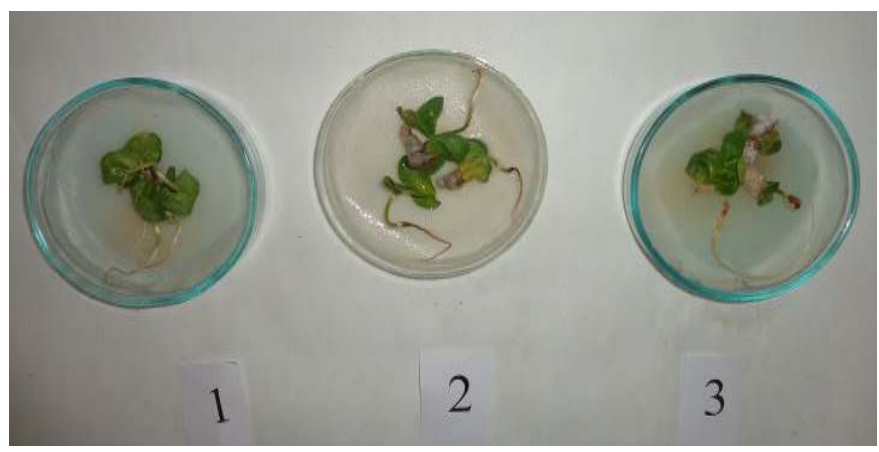

(c)

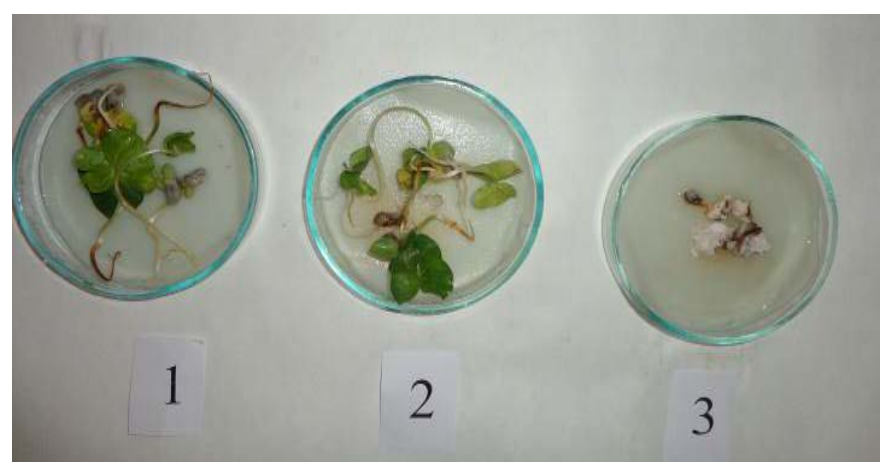

(d)

Explanation: 1. Control; 2. Verticillium dahliae, 3. Fusarium oxysporum f.sp. vasinfectum

Figure 5. Impetuosity for mycotoxin backcross hybrid combinations. (a) $\mathrm{F}_{3} \mathrm{~B}_{1} \mathrm{C}$ Namangan $77 \times$ [Namangan $77 \times($ ssp. obtusifolium var. indicum $\times$ G. australe)] "Family 2"; (b) $\mathrm{F}_{3} \mathrm{~B}_{1} \mathrm{C}$ [Namangan $77 \times($ ssp. obtusifolium var. indicum $\times$ G. australe $\left.)\right] \times$ Namangan 77 "Family 1"; (c) $\mathrm{F}_{3} \mathrm{~B}_{1} \mathrm{C}$ Kelajaк $\times$ [Kelajak $\times$ (ssp. nanking (ok толали) $\times$ G. nelsonii) $]$ "Family 3"; (d) $\mathrm{F}_{3} \mathrm{~B}_{1} \mathrm{C}$ [Кelajaк $\times$ (ssp. nanking (ok толали) $\times$ G. nelsonii) $] \times$ Кеlaјак "Family 4". 
However, Fusarium oxysporum f.sp. vasinfectum was unstable in "Family 1", "Family 2" and "Family 3". $\mathrm{F}_{3} \mathrm{~B}_{1} \mathrm{C}$ [Namangan $77 \times$ (ssp. obtusifolium var. indicum $\times$ G. australe)] $\times$ Namangan 77 was presented in the strong "Family 1", "Family 2" in Verticillium dahliae's strong resistance. In the "Family 3", Verticillium dahliae was found to be less tolerant (33.3\% of seeds did not grow) in the backcross hybrid combination of $\mathrm{F}_{3} \mathrm{~B}_{1} \mathrm{C}$ [Namangan $77 \times$ (ssp. obtusifolium var. indicum $\times$ G. australe) $] \times$ Namangan 77. $\mathrm{F}_{3} \mathrm{~B}_{1} \mathrm{C}$ [Namangan $77 \times$ (ssp. obtusifolium var indicum $\times G$. australe) $] \times$ Namangan 77 combustion combination "Family 4" Verticillium dahliae, Fusarium oxysporum f.sp. vasinfectum the degree of resistance of mycotoxins separated from micromycetes to germliness of plant seeds. $\mathrm{F}_{3} \mathrm{~B}_{1} \mathrm{C}$ [Namangan $77 \times$ (ssp. obtusifolium var. indicum $\times$ G. australe)] $\times$ Namangan 77 was found to be high in tolerance to Fusarium oxysporum f.sp. vasinfectum in "Family 2" and "Family 3" backcross hybrid combination. $\mathrm{F}_{3} \mathrm{~B}_{1} \mathrm{C}$ [Namangan $77 \times($ ssp. obtusifolium var. indicum $\times$ G. australe $\left.)\right] \times$ Namangan 77 backcross hybrid combination with "Family 1" Fusarium oxysporum f.sp. vasinfectum was shown to be strong (Figure 5(b)).

$\mathrm{F}_{3} \mathrm{~B}_{1} \mathrm{C}$ Kelajak $\times[$ Kelajak $\times($ ssp. nanking $($ white fiber $) \times G$. nelsonii) $)]$ as a result of mycotoxins isolated from the Verticillium dahliae microcrycine in the family "Family 1", "Family 2", "Family 4" 25.0\% - 50.0\% of seeds were sown. $\mathrm{F}_{3} \mathrm{~B}_{1} \mathrm{C}$ Kelajak $\times$ [Kelajak $\times$ (ssp. nanking (white fiber) $\times$ G. nelsonii) $]$ was found to be high in tolerance to the Verticillium dahliae in "Family 3" (Figure 5(c)). $\mathrm{F}_{3} \mathrm{~B}_{1} \mathrm{C}$ Kelajak $\times[$ Kelajak $\times($ ssp. nanking $($ white fiber $) \times G$. nelsonii) $]$ as a result of the effects of mycotoxins separated from Fusarium oxysporum f.sp. vasinfectum in "Family 2", "Family 3", "Family 4" 95.0\% of seeds were sown. It was found that $75.0 \%$ of the seeds did not grow due to $\mathrm{F}_{3} \mathrm{~B}_{1} \mathrm{C}$ Kelajak $\times$ [Kelajak $\times$ (ssp. nanking (white fiber) $\times G$. nelsonii)] with the effect of the combination of bacterial hybrid "Family 1" Fusarium oxysporum f.sp. vasinfectum. $\mathrm{F}_{3} \mathrm{~B}_{1} \mathrm{C}[\mathrm{Ke}-$ lajak $\times$ (ssp. nanking (white fiber) $\times$ G. nelsonii)] In the family "Family 1" and "Family 2" combination of future bacterial bacteria, Verticillium dahliae was found to be $25.0 \%-50.0 \%$, And in "Family 3", it was noted that seeds were generally unchanged. $\mathrm{F}_{3} \mathrm{~B}_{1} \mathrm{C}$ [Kelajak $\times$ (ssp. nanking (white fiber) $\times$ G. nelsonii)] in "Family 4", the combination of anticoagulant hybridis with Verticillium dahliae was found to be highly tolerant (Figure 5(d)). $\mathrm{F}_{3} \mathrm{~B}_{1} \mathrm{C}$ [Kelajak (ssp. nanking (white fiber) G.nelsonii)] $\times$ Kelajak backcross hybrid combinations Fusarium oxysporum f.sp. vasinfectum mycotoxins are resistant. In particular, "Family 1" and "Family 2" were affected by mycotoxins $25.0 \%$, "Family 3" and "Family 4" were strongly endangered.

The $\mathrm{F}_{4} \mathrm{C}$ Namangan $77 \times($ ssp. obtusifolium var. indicum $\times$ G.australe $)$ was recorded in the "Family 2" and "Family 3" combination of hybrid combination with Verticillium dahliae high reliability (95.0\%). As a result of mycotoxins isolated from Fusarium oxysporum f.sp. vasinfectum micro metracycry, 80.0\% $90.0 \%$ of the seeds were found (Figure 6). $\mathrm{F}_{4} \mathrm{C}$ Namangan $77 \times$ (ssp. obtusifolium var. indicum $\times G$. australe) resulted in $70.0 \%$ of mycotoxins isolated 


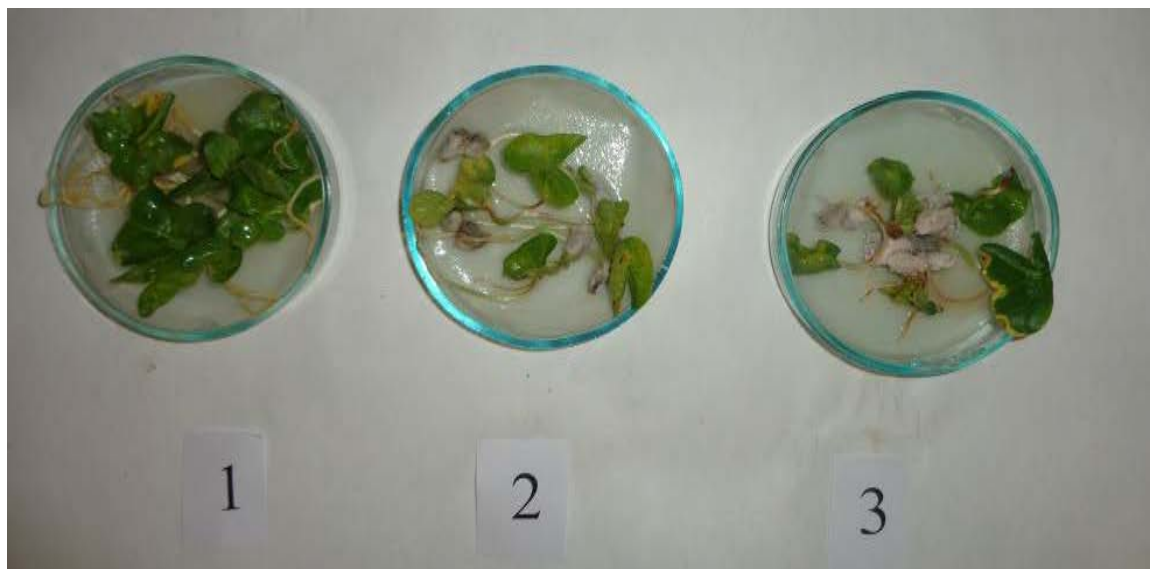

Explanation: 1. Control; 2. Verticillium dahliae, 3. Fusarium oxysporum f.sp. vasinfectum

Figure 6. $\mathrm{F}_{4} \mathrm{C}$ Namangan $77 \times($ ssp. obtusifolium var. indicum $\times$ G. australe $)$ "Family 2 ".

from microparticles microcrystals "Family 1" and "Family 4" in Verticillium dahliae and Fusarium oxysporum f.sp. vasinfectum, seeds were sown. $\mathrm{F}_{4} \mathrm{C}$ Kelajak $\times$ (ssp. nanking (white fiber) G. nelsonii) was found to be moderately resistant to the Verticillium dahliae, Fusarium oxysporum f.sp. vasinfectum mycotoxins in the "Family 1" combination of hybrids.

The $\mathrm{F}_{4} \mathrm{C}$ Kelajak $\times$ (ssp. nanking (white fiber) G. nelsonii) was recorded in Verticillium dahliae and Fusarium oxysporum f.sp. vasinfectum at $90.0 \%$ in family "Family 2". $\mathrm{F}_{4} \mathrm{C}$ Kelajak $\times$ (ssp. nanking (white fiber) G. nelsonii) "Family 3 " showed that $95.0 \%$ seeds were affected by the Verticillium dahliae mycotoxin effect. As a result of the effects of mycotoxins separated from the Fusarium oxysporum f.sp. vasinfectum microcrysta, $80.0 \%$ were sown. The $\mathrm{F}_{4} \mathrm{C}$ Kelajak $\times$ (ssp. nanking (white fiber) G. nelsonii) hybrid combination was found to be 96.0\% in Verticillium dahliae, Fusarium oxysporum f.sp. vasinfectum with a combination of hybrid combination (Figure 7).

Thus, according to the analysis of the degree of effectiveness of mycotoxin sulphide seeds on microorganisms of Verticillium dahliae, Fusarium oxysporum f.sp. vasinfectum microscitres, new recombinant hybrid forms were found to be comparable to $\mathrm{F}_{2} \mathrm{C}$ hybrid combination in $\mathrm{F}_{4} \mathrm{C}$ hybrid combination combinations, Verticillium dahliae, Fusarium oxysporum f.sp. vasinfectum, how much $\mathrm{F}_{4} \mathrm{C}$ demonstrates the presence of polymeric nature of the dominant alleles of the genes involved in the growth of the gene in the generation. $\mathrm{F}_{3} \mathrm{~B}_{1} \mathrm{C}$ Namangan 77 $\times$ [Namangan $77 \times($ ssp. obtusifolium var. indicum $\times$ G. australe)] "Family 2", $\mathrm{F}_{3} \mathrm{~B}_{1} \mathrm{C}$ [Kelajak $\times$ (ssp. nanking (white fiber) G. nelsonii)] $\times$ Kelajak "Family 1", $\mathrm{F}_{4} \mathrm{C}$ Namangan $77 \times$ (ssp. obtusifolium var. indicum $\times$ G. australe) "Family 2", "Family 3", $\mathrm{F}_{4} \mathrm{C}$ Kelajak $\times$ (ssp. nanking (white fiber) G. nelsonii) "Family 3" Verticillium dahliae micromoxetine mycotoxins tolerance. $\mathrm{F}_{3} \mathrm{~B}_{1} \mathrm{C}$ [Namangan 77 $\times$ (ssp. obtusifolium var. indicum $\times$ G. australe) $] \times$ Namangan 77 "Family 2", "Family 3", $\mathrm{F}_{3} \mathrm{~B}_{1} \mathrm{C}$ Kelajak $\times$ [Kelajak $\times$ (ssp. nanking $($ white fiber $) \times$ G. nelsonii) "Family 2", "Family 4", $\mathrm{F}_{4} \mathrm{C}$ Kelajak $\times$ (ssp. nanking (white fiber) G. nelsonii)] mycotoxins of Fusarium oxysporum f.sp. vasinfectum microscopy in "Family 2" 


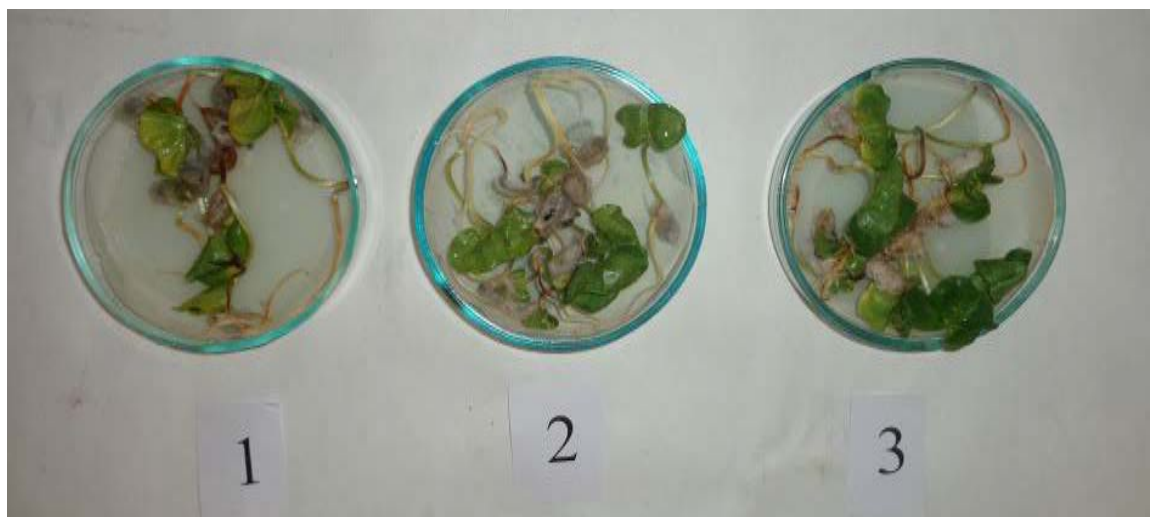

Explanation: 1. Control; 2. Verticillium dahliae, 3. Fusarium oxysporum f.sp. vasinfectum

Figure 7. $\mathrm{F}_{4} \mathrm{C}$ Kelajaк $\times($ ssp. nanking $($ white fiber $) \times$ G.nelsonii $)$ "Family 4".

are strongly resistant to germliness of plant seeds was observed. $\mathrm{F}_{3} \mathrm{~B}_{1} \mathrm{C}$ [Namangan $77 \times$ (ssp. obtusifolium var. indicum $\times$ G.australe) $] \times$ Namangan 77 "Family 4 ", $\mathrm{F}_{3} \mathrm{~B}_{1} \mathrm{C}$ Kelajak $\times[$ Kelajak $\times$ (ssp. nanking (white fiber) G. nelsonii)] "Family 3", $\mathrm{F}_{4} \mathrm{C}$ Kelajak $\times($ ssp. nanking (white fiber) $\times$ G. nelsonii) hybrid combinations Verticillium dahliae, Fusarium oxysporum f.sp. vasinfectum, have been found to have high rates of mycotoxin susceptibility to microbial seeds. The new artificial complicated hybrid forms combine the potential for resistance to the disease in their Kario-Plazma on agricultural diseases, making a huge contribution to the selection of new varieties and the effectiveness of selection as a result of the use of genetic selective research in genetic-selective studies on the improvement of economic characteristics.

\section{References}

[1] Berestetsky, O.A. (1978) Phytotoxins of Soil Microorganisms and Their Ecological Role. Phytotoxic Properties of Soil Microorganisms. All-Union Institute of Agricultural Microbiology, Leningrad, 7-30.

[2] Sheraliev, A.Sh. (1977) Phytotoxic Properties of Fungi of the Genus Fusarium Lk. on the Mulberry in Uzbekistan. Microbiological Journal, 5, 12-16. (In Ukrainian)

[3] Sherimbetov, A.G. (2016) Fusarium turkumiga mansub zambuuruu lardan azhratilgan mycotoxin larinizing y̆siliklar uruinning uznchanaligiga tacиsirini y̆rganis. Uzbekiston agrar fani habarnomasi, 3, 80-85.

[4] Abdullaev, A.A. (2003) The Importance of Cotton Gene Pool. Bulletin of Agrarian Science of Uzbekistan, 12, 52-56.

[5] Arutyunova, L.G. (1960) Interspecific Hybridization in Genus Gossypium L. Questions of Genetics of Selection and Seed Production of Cotton. Sat \& Art Antenas, Camaçari, 71 .

[6] Babamuratov, H. (1976) Inheritance of Some Morphological and Economic Features of Tri-Genomic Cotton Hybrids. Problems of Genetics, Selection and Seed-Growing of Cotton and Alfalfa, 13, 14-18.

[7] Babamuratov, H. (1980) Method of Backcrossing in Remote and Interspecific Hybridization of Cotton. Proceedings of the All-Union Research Institute of Selection and Seed Cotton, 18, 32. 
[8] Mahmudov, T.K., Sadykhova, L.D. and Mamedov, F.Kh. (1982) Remote Hybridization in Combination with Experimental Polyploidy and Its Use in Cotton Breeding. Bulletin of Agriculture. Sciences, 1, 22-26.

[9] Makhmudov, T.K. and Eldarov, S.I. (1990) Hexaploid G. hirsutum $\times$ G. raimondii and Its Use in Practical Cotton Breeding. Bulletin of Agricultural Sciences, 5, 29-32.

[10] Mendes, A.J.T. (1940) Polyploid Cotton Obtained through Use of Colchicines. I. Cytological Observation in Octoploid Gossypium hirsutum. Bot. Caz, 102, 287-294.

[11] Choi-Pheng, Y. and Wray, B. (1984) Resistant germplasm in Gossypium L spesies and related plants to Rotylenchulus reniformis. Journal of Nematology, 16, 146-153.

[12] Mayer, F.M. (1938) Первые амфидиплоиды и другие полиплоидные межвидовые гибриды у Gossypium L. Советский хлопок, 2, 46-53.

[13] Liu, Q., Chen, Y., Chen, Y., Wang, Y., Chen, J., Zhang, T., et al. (2015) A New Synthetic Allotetraploid $\left(\mathrm{A}_{1} \mathrm{~A}_{1} \mathrm{G}_{2} \mathrm{G}_{2}\right)$ between Gossypium herbaceum and G. australe: Bridging for Simultaneously Transferring Favorable Genes from These Two Diploid Species into Upland Cotton. PLoS ONE, 10, 1-16.

[14] Chen, Y., Wang, Y., Wang, K., Zhu, X., Guo, W., Zhang, T. and Zhou, B. (2014) Construction of a Complete Set of Alien Chromosome Addition Lines from Gossypium australe in Gossypium hirsutum: Morphological, Cytological, and Genotypic Characterization. Theoretical and Applied Genetics, 127, 1105-1121. https://doi.org/10.1007/s00122-014-2283-1

[15] Anh, L.D. (1995) Factors of Resistance of Wild and Cultivated Representatives of the Genus Gossypium L. to Cotton Flax (Aphis gossupii Glov.): Author's Abstract. Diss. ... Cand. Biol. Sciences, Tashkent, 23 p.

[16] Luo, J., Cui, J. and Xin, H. (2012) Relationship between the Contents of Cellulose and Lignin in Cotton Leaf and Their Resistance to Apolygus lucorum. Journal of Northwest $A$ \& F University (Natural Science Edition), 40, 81-85.

[17] Akhtar, K.P., Haidar, S., Khan, M.K.R., Ahmad, M., Sarwar, N., Murtaza, M.A., et al. (2010) Evaluation of Gossypium Species for Resistance to Cotton Leaf Curl Burewala Virus. Annals of Applied Biology, 157, 135-147.

[18] Ma, Z.Y., Wang, X.F., Zhang, G.Y., Li, X.H., Liu, S.Q., Wu, L.Q., et al. (1997) Study on the Pathogenicity of Verticillium dahliae of Cotton in Hebei Province. Acta Gossypii Sinica, 9, 15-20.

[19] Ma, Z.Y., Wang, X.F., Zhang, G.Y., Liu, Z.G., Li, X.H., Liu, S.Q., et al. (1998) Distribution of the Isolates of Different Virulence Groups of Verticillium dahliae in Hebei Cotton Growing Areas. Journal of Agricultural University of Hebei, 21, 1-6.

[20] Zhang, L., Li, F., Liu, C., Zhang, C. and Wu, Z. (2010) Isolation and Analysis of Drought-Related Genefrom Cotton (Gossypium arboreum L.) SSH Library. Cotton Science, 22, 110-114.

[21] Kanash, S.S. (1932) Interspecific Hybridization within the Different-Chromosome Species of Cotton. Tashkent, Saogiz, $56 \mathrm{p}$.

[22] Kanash, S.S. (1936) Interspecific Hybridization within the Different-Chromosomal Species of Cotton. Collection: Brief Contents and Direction of Research Works of the TsSU SoyuzNIKHI, Tashkent, 42-47.

[23] Ansingkar, A.S., Khade, P.P., Borikar, S.T. and Bhosle, S.S. (2004) Altering G. hirsutum Cotton at Cellular Level to Impart Multiple Sucking Pest Resistance through Interspecific Hybridization. In: Khadi, B.M., Katageri, I.S., Patil, S.S., Vamadevaiah, H.M., Patil, B.R. and Manjula, S.M., Eds., Proceedings of International Symposium on "Strategies for Sustainable Cotton Production-A Global Vision, 1, Crop Im- 
provement, University of Agricultural Sciences, Dharwad, 101-103.

[24] Sirojidinov, B.A. (2017) Phylogenetic Interrelations of the Australian and Indochinense Cotton Species: Author's Abstract. Bio. Sciences, Tashkent, 40 p. 